\title{
毛乌素沙地油蓄种群点格局分析
}

\author{
杨洪晓 ${ }^{1}$ 张金屯 ${ }^{*}$ 吴 波 $^{2}$ 李晓松 ${ }^{3}$ 张友炎 ${ }^{2}$ \\ (1 北京师范大学生命科学学院, 北京 100875) (2 中国林业科学研究院林业研究所, 北京 100091) \\ (3 中国林业科学研究院资源信息研究所, 北京 100091)
}

摘 要 油蒿 (Artemisia ordosica) 是我国北方农牧交错带的重要固沙植物, 研究其种群格局对理解种群生态过程和 改善流沙治理技术具有重要意义。点格局分析法是 20 世纪末发展起来的多尺度空间格局分析方法。通过研究油 蒿种群的点格局 发现油蒿种群的空间分布格局和空间关联性同空间尺度、植株形体大小以及生境 3 种因素有密 切联系。在较小的空间尺度上, 油蕎种群倾向于非随机分布 (集群分布比均匀分布常见), 个体间有较强的空间关 联(正关联比负关联常见); 当空间尺度大于临界值后, 油蒿种群倾向于服从随机分布, 同时种群的空间关联性减 弱。幼小油蒿植株具有明显的集群分布趋势, 高大植株则表现出聚集强度的降低趋势; 形体大小的差异越大, 植株 间的正关联关系越弱, 或者负关联关系越强。与固定沙地相比, 半固定沙地油蒿种群的集群分布现象更加明显, 同 时种群的空间正关联关系更强。研究结果表明, 当通过移栽油蒿成体治理流动沙地时, 应尽量将其栽种成集群分 布而非均匀分布的形式，以提高植株成活率。

关键词 油蓄 点格局分析 分布格局 空间关联 固沙作用

\section{POINT PATTERN ANALYSIS OF ARTEMISIA ORDOSICA POPULATION IN THE MU US SANDY LAND}

\author{
YANG Hong-Xiao $^{1}$ ZHANG Jin-Tun ${ }^{1 *}$ WU Bo ${ }^{2}$ LI Xiao-Song ${ }^{3}$ and ZHANG You-Yan ${ }^{2}$ \\ (1 College of Life Sciences , Beijing Normal University , Beijing 100875 , China) \\ (2 Research Institute of Forestry, Chinese Academy of Forestry , Beijing 100091, China) \\ (3 Institute of Forest Resource Information Techniques, Chinese Academy of Forestry , Beijing 100091 , China)
}

\begin{abstract}
Background and Aims Artemisia ordosica is an important sand fixation plant in north China , whereas few studies were conducted on its population patterns. Population patterns of this species are therefore studied, and the aims are mainly :1) how spatial scales are related to population patterns ,2) whether plant sizes effect population patterns, and 3) what roles environments play in the process of pattern formation?
\end{abstract}

Methods Two plots in the size of $50 \mathrm{~m} \times 50 \mathrm{~m}$ were established in the Mu Us sandy land, and all the A. ordosica plants were recorded and mapped. After that, the data were analyzed with the method of point pattern analysis.

Key results On the scales less than certain critical points, the population is usually characterized by nonrandom distribution (being clumped or regular), and their spatial association is considerably strong, but may be positive or negative. By contrast, if the scales enlarge beyond these points , the plants will disperse randomly , and their spatial association will loosen greatly. Small individuals are more likely to follow clumped distribution than those big ones. The spatial association between plants will turn from positive to negative if their size differences are enlarged increasingly. Unlike the $A$. ordosica plants growing in fixed sandy land, those plants in semi-fixed sandy land are easier to follow clumped distribution and associate with each other positively.

Conclusions Population patterns and spatial association of $A$. ordosica depend on spatial scales , individual sizes and environments, and so do their intra-specific relationships. If $A$. ordosica plants are transplanted into shifting sandy land for sand control and vegetation restoration , they ought to be arranged in the form of clumped distribution rather than regular distribution.

Key words Artemisia ordosica, Point pattern analysis, Distribution pattern , Spatial association , Sand fixation 
空间格局 (Spatial pattern) 是植物种群的基本特 征, 研究植物种群的空间格局有助于认识它们的生 态过程以及它们与生境的相互关系 (Greig Smith , 1983 ; 阳含熙等, 1985 ; Dale, 1999)。其中, 判定种 群的空间分布类型 (Spatial distribution) 和空间关联 性 (Spatial association) 是空间格局研究的两个主要内 容(张金屯, 2004)。种群的空间分布类型与空间关 联性是一致的, 它们是种群生态关系在空间格局上 的两种表现形式。植物种群的空间分布有 3 种基本 类型:集群分布 (Clumped distribution)、随机分布 (Random distribution) 和均匀分布 (Regular distribution）相应地, 种群的空间关联有 3 种基本方式: 空 间正关联 (Positive spatial association)、空间无关联 (Independent spatial association) 和空间负关联 (Negative spatial association) (张金屯, 2004)。集群分布和 空间正关联体现了种群内部正向 (相互有利) 的生态 关系均匀分布和空间负关联反映了种群内部负向 (相互排斥) 的生态关系, 随机分布和空间无关联则 意味着种群内部没有明确的生态关系 (Philips \& Macmahon , 1981 ; Kenkel , 1988 ; Brission \& Reynolds , 1994 ; Manuel , 2000)。

传统方法依靠单一尺度 (固定大小) 的若干样方 研究植物种群的空间分布格局, 只能对特定空间尺 度上的分布格局得到具体认识 (张金屯, 1998 ; 李 先琨等, 2003 ; 张金屯, 2004)。然而, 空间格局对 空间尺度具有很强的依赖性, 植物种群在某些尺度 上可能服从集群分布, 在其它尺度上却可能改变为 随机分布或均匀分布 (Greig Smith，1983; 阳含熙等， 1985)。显然，使用传统方法在特定空间尺度上得到 的认识不能推广或应用到其它空间尺度上去。Ripley (1977)提出了能以由小到大的一系列连续尺度 分析点状物体平面分布格局的方法, 称点格局分析 法(Point pattern analysis)。由于这种方法克服了传 统方法只能分析单一尺度空间分布格局的缺点, 很 快被发展并应用到植物肿群多尺度空间分布格局和 两个物种之间多尺度空间关联的研究中 (Diggle, 1983 ; Andersen , 1992 ; Dale \& Powell , 2001 ; 张金屯， 2004)。从 20 世纪 90 年代, 我国学者陆续运用点格 局分析法研究森林或草地物种的种群分布格局或空 间关联性, 得以很好地从多尺度上理解植物种群的 生态学特性或过程(张金屯, 1998 ; 汤孟平等, 2003 ; 刘振国和李镇清，2004; 张金屯和孟东平， 2004)。

油蒿 (Artemisia ordosica) 是我国温带沙漠的优良
固沙半灌木, 分布于以鄂尔多斯高原为中心的温带 半干旱沙区(杨洪晓等, 2004)。许多学者研究了油 蒿的形态结构、生理特征和繁殖特性以及这些特征 与沙地环境的关系, 还对油蒿种群或群落结构进行 了探讨(刘家琼等，1988; 王刚等，1995; 王刚和梁 学功, 1995 ; 王庆锁等, 1997 ; Huang \& Gutterman, 1999 ; 郭柯,2000; 于云江等，2002)。然而, 目前对 油蓠种群空间格局的研究仍不够充分, 更没有见到 将空间尺度、种群大小结构 (形体大小不同的各等级 的相对个体数量) 和生境同种群空间格局联系起来 进行研究的先例。鉴于此, 我们试图运用点格局分 析方法在鄂尔多斯高原的毛乌素沙地研究空间尺 度、种群大小结构和生境 3 种因素与油蓄种群的空 间分布类型或空间关联性的关系，以期从空间格局 这个基本特征认识油蒿种群的某些生态学性质或过 程, 并为合理利用油蒿治理我国北方的沙化土地提 供参考依据。

\section{1 研究材料与方法}

\section{1 取样}

毛乌素沙地是我国温带半干旱地区的重要沙 地, 位于内蒙古、陕西、宁夏的交界地带, 地理位置为 $37^{\circ} 27.5^{\prime} \sim 39^{\circ} 22.5^{\prime} \mathrm{N}$ 和 $107^{\circ} 20^{\prime} \sim 111^{\circ} 30^{\prime} \mathrm{E}$, 是鄂尔 多斯高原向黄土高原的过渡地区(张新时, 1994)。 以油蓄为建群种的沙生植被约占毛乌素沙地总面积 的 30\%以上, 油蒿群落是毛乌素沙地面积最大的群 系, 油蓠作为最重要的乡土植物在防沙固沙和维持 区域生态系统健康等方面发挥了重要作用 (北京大 学地理系, 1983 ; 郭柯, 2000)。

2004 年 8 月，在毛乌素沙地的乌审旗图克苏木 开展野外调查。在半固定沙地和固定沙地分别设立 两块 $50 \mathrm{~m} \times 50 \mathrm{~m}$ 的典型样地, 油蕎是样地植物群落 的单优建群种。在半固定沙地的样地中油蓄盖度在 $10 \%$ 以上 其它植物种类很少; 在固定沙地的样地中 油蕎盖度约 $40 \%$, 其它物种的盖度不足 $10 \%$ 。测量 并记录每株油蓄的长、短两个冠幅和地上部分的高 度 相对于设定原点 (样地某顶点) 的空间位置 ( $X 、 Y$ 坐标值)。

因为植株体积是反映其形体大小的重要指标, 所以依据植株体积将油蓄划分为不同的大小级。植 株体积的计算使用了长短两个冠幅和地上部分的高 度三者的乘积。因为不同植株的相对大小取决于植 株的长、短冠幅和高度三者的乘积而不是常数 $\pi$,所 以植株体积的计算省略了这个常数。依据植株体积 
的立方根 $(d)$ 将油蒿划分为 4 个大小级 :大小级 $1, d$ $\leq 25 \mathrm{~cm}$;大小级 $2,25 \mathrm{~cm}<d \leq 50 \mathrm{~cm}$;大小级 3,50 $\mathrm{cm}<d \leq 75 \mathrm{~cm}$;大小级 $4, d>75 \mathrm{~cm}$ 。

在半固定沙地的样地中有 1960 株油蒿, 其中， 大小级 1 有 948 株, 大小级 2 有 493 株, 大小级 3 有 361 株, 大小级 4 有 158 株; 在固定沙地的样地中有 2341 株油蒿, 其中, 大小级 1 有 39 株, 大小级 2 有 501 株, 大小级 3 有 965 株, 大小级 4 有 836 株。

\section{2 格局分析}

计算半固定沙地和固定沙地样地油蒿种群以及 它们每个大小级的 $\check{K}(t)$ 值, $\check{K}(t)$ 可反映相应群体 在空间尺度 $t$ 上的分布格局 (Dale \& Powell, 2001 ; 张金屯, 2004 ; 张金屯和孟东平, 2004)。 $\check{K}(t)$ 值的 计算公式如下：

$$
\check{K}(t)=\left(\frac{A}{n^{2}} \sum_{i=1}^{n} \sum_{j=1}^{n} \frac{1}{W_{i j}} I_{t}\left(u_{i j}\right) \quad(i \neq j)\right.
$$

其中 , $A$ 表示样地面积 ; $n$ 表示半固定沙地或固 定沙地样地油蓄种群的总体或者它们每个大小级的 个体总数; $u_{i j}$ 表示第 $i$ 株油蒿和第 $j$ 株油蒿之间的距 离, $t$ 表示空间尺度, 当 $u_{i j} \leqslant t$ 时, $I_{t}\left(u_{i j}\right)=1$, 反之, $I_{t}\left(u_{i j}\right)=0 ; W_{i j}$ 为以点 $i$ 为圆心、以 $u_{i j}$ 为半径的圆落 在面积 $A$ 中的弧长与整个圆周的比值, 可校正边界 效应引起的误差。

计算半固定沙地或固定沙地样地油蕎种群每两 个大小级之间的 $\check{V}(t)$ 值, $\breve{V}(t)$ 反映大小级 $p$ 和 $q$ 在 空间尺度 $t$ 上的空间关联性 :

$$
\check{V}(t)=\left(\frac{A}{n_{p} n_{q}} \sum_{i=1}^{n_{p}} \sum_{j=1}^{n_{q}} \frac{1}{W_{i j}} I_{t}\left(u_{i j}\right) \quad(i \neq j)\right.
$$

其中, $n_{p}$ 和 $n_{p}$ 分别表示大小级 $p$ 和 $q$ 的个体

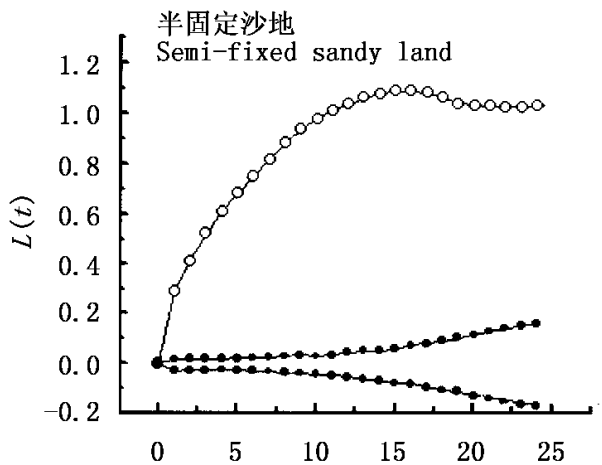

数 $; i$ 代表大小级 $p$ 的第 $i$ 株油蒿,$j$ 代表大小级 $q$ 的 第 $j$ 株油蓠; $u_{i j}, W_{i j}$ 和 $A$ 的含义同公式(1)。

根据 Kenkel (1988)的建议 将公式(1)简化为公 式(3)：

$$
L(t)=\sqrt{\check{K}(t) / \pi}-\mathrm{t}
$$

当 $L(t)>0$ 相应群体在尺度 $t$ 服从集群分布; 当 $L(t)=0$, 相应群体在尺度 $t$ 服从随机分布; 当 $L(t)<0$ 相应群体在尺度 $t$ 服从均匀分布。

同理，公式(2)可转化为公式(4)：

$$
M(t)=\sqrt{\check{V}(t) / \pi}-\mathrm{t}
$$

当 $M(t)>0$,大小级 $p$ 和 $q$ 在尺度 $t$ 具有正关 联; 当 $M(t)=0$,大小级 $p$ 和 $q$ 在尺度 $t$ 没有关联； 当 $M(t)<0$,大小级 $p$ 和 $q$ 在尺度 $t$ 具有负关联。

为检验 $L(t)$ 或 $M(t)$ 偏离零值的显著程度, Manly (1997) 提出用 Monte-Carlo 随机模拟方法拟合 它们 $95 \%$ 的置信区间。

数据分析过程通过生态学软件包 ADE-4 和 ORIGIN7.0 完成。采用的空间尺度由 0 一直增加到 $24 \mathrm{~m}$,步长为 $1 \mathrm{~m}$;Monte-Carlo 随机模拟的数目为 200 次 得到 $L(t)$ 或 $M(t)$ 由上下两条包迹线围成的 $95 \%$ 置信区间。

\section{2 结果与分析}

\section{1 油蓄种群的空间分布}

在小于 $24 \mathrm{~m}$ 的一系列空间尺度上,两种条件下 的油蒿种群主要呈集群分布，但固定沙地油蒿种群 的聚集强度低于半固定沙地的油蒿种群, 甚至固定 沙地油蒿种群在 $1 \mathrm{~m}$ 尺度上表现出均匀分布 (图 1)。

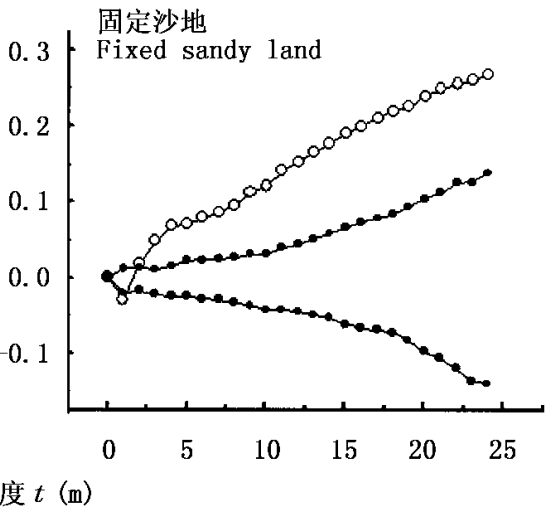

$\longrightarrow L(t) \quad \longrightarrow$ 上下包迹线 Upper and lower envelopes (95\%)

图 1 半固定沙地和固定沙地油蒿种群的空间分布格局

Fig.1 Spatial patterns of Artemisia ordosica population in semi-fixed or fixed sandy land 


\section{2 油蒿种群不同大小级的空间分布}

对于半固定沙地的油蓠种群, 在 $24 \mathrm{~m}$ 以内的空 间尺度上 4 个大小级的值大于 0 且超出包迹线的 现象较普遍, 表明各大小级主要呈现集群分布, 其中 以大小级 1 的 $L(t)$ 最大, 即聚集强度最大。在大于 $22 \mathrm{~m}$ 的尺度上大小级 2 的 $L(t)$ 值已落入包迹线围 成的置信区间, 表明大小级 2 的空间分布类型变为 了随机分布。在大于 $17 \mathrm{~m}$ 的尺度上, 大小级 3 的空 间分布类型也变为随机分布 (图 2)。另外, 在大于 二十几米的某个尺度上, 大小级 1 和 4 的空间分布
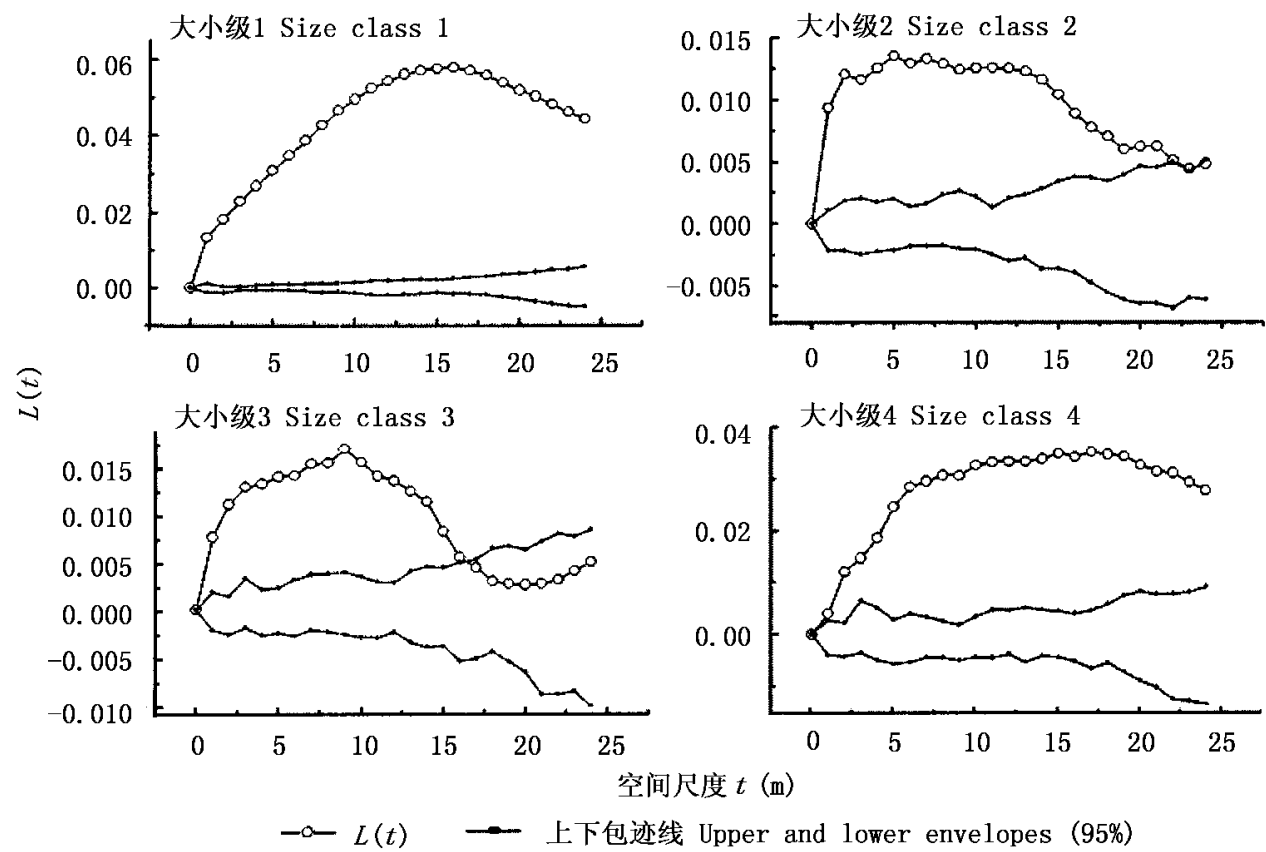

空间尺度 $t(\mathrm{~m})$

图 2 半固定沙地油蒿种群 4 个大小级的空间分布格局

Fig.2 Spatial patterns of four size classes of Artemisia ordosica population in semi-fixed sandy land

\section{3 油蒿种群不同大小级的空间关联}

对于半固定沙地的油蒿种群, 在较小的尺度上， $M(t)$ 值接近或超出包迹线的机会或程度更大，表明 油蓠种群各大小级间的空间关联 (包括正负两种关 联)主要表现在小尺度上;随空间尺度的增加, $M(t)$ 曲线逐渐被上下包迹线包围, 空间关联变得很微弱 (图 4)。就它们在较小尺度上的空间关联性而言， 如果两个大小级的形体大小程度越接近,它们的 $M(t)$ 值就越容易高于上包迹线, 表现出较强的正关 联关系; 汉之, $M(t)$ 值就容易低于下包迹线, 表现出 负关联关系。

在固定沙地上油蓠种群不同大小级之间的空间 关联性与在半固定沙地上类似，即: 固定沙地油蒿种
群不同大小级之间的空间关联性主要表现在小尺度 上, 随空间尺度的增加, 各大小级之间的关联性减 弱; 当在较小的空间尺度上,形体大小程度越接近的 大小级之间越容易产生正关联现象，反之，容易产生 负关联现象 (图 5)。然而, 与在半固定沙地上相比, 固定沙地相应大小级的空间关联性发生了变化，即： 正关联程度表现出不同程度的降低, 或者如果两个 大小级在半固定沙地上是负关联, 那么它们在固定 沙地上的负关联会更强。其中, 以大小级 3 和 4 之 间空间关联性的变化最显著。

\section{3 讨论与结论}

\section{1 空间尺度与空间格局的联系}



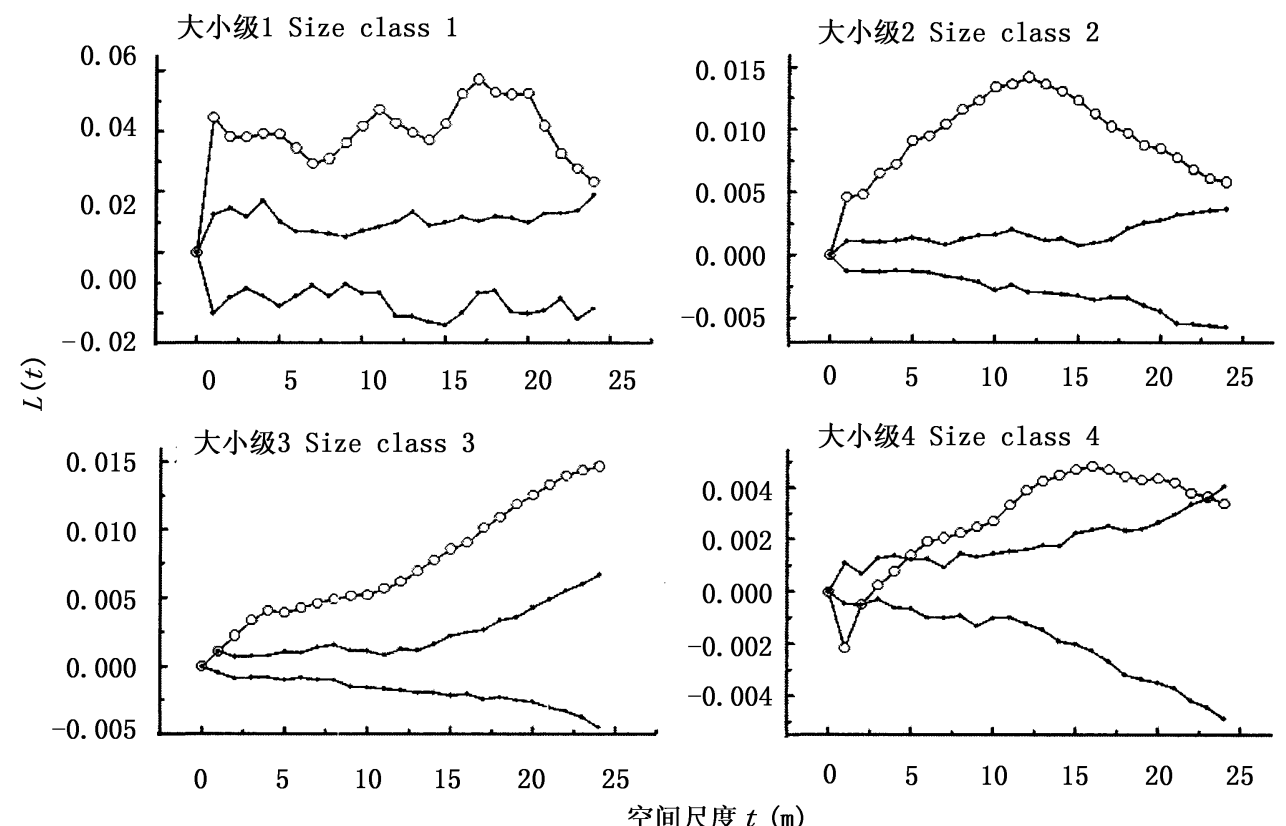

空间尺度 $t(\mathrm{~m})$

一 $L(t) \rightarrow$ 上下包迹线 Upper and lower envelopes (95\%)

图 3 固定沙地油蒿种群 4 个大小级的空间分布格局

Fig.3 Spatial patterns of four size classes of Artemisia ordosica population in fixed sandy land
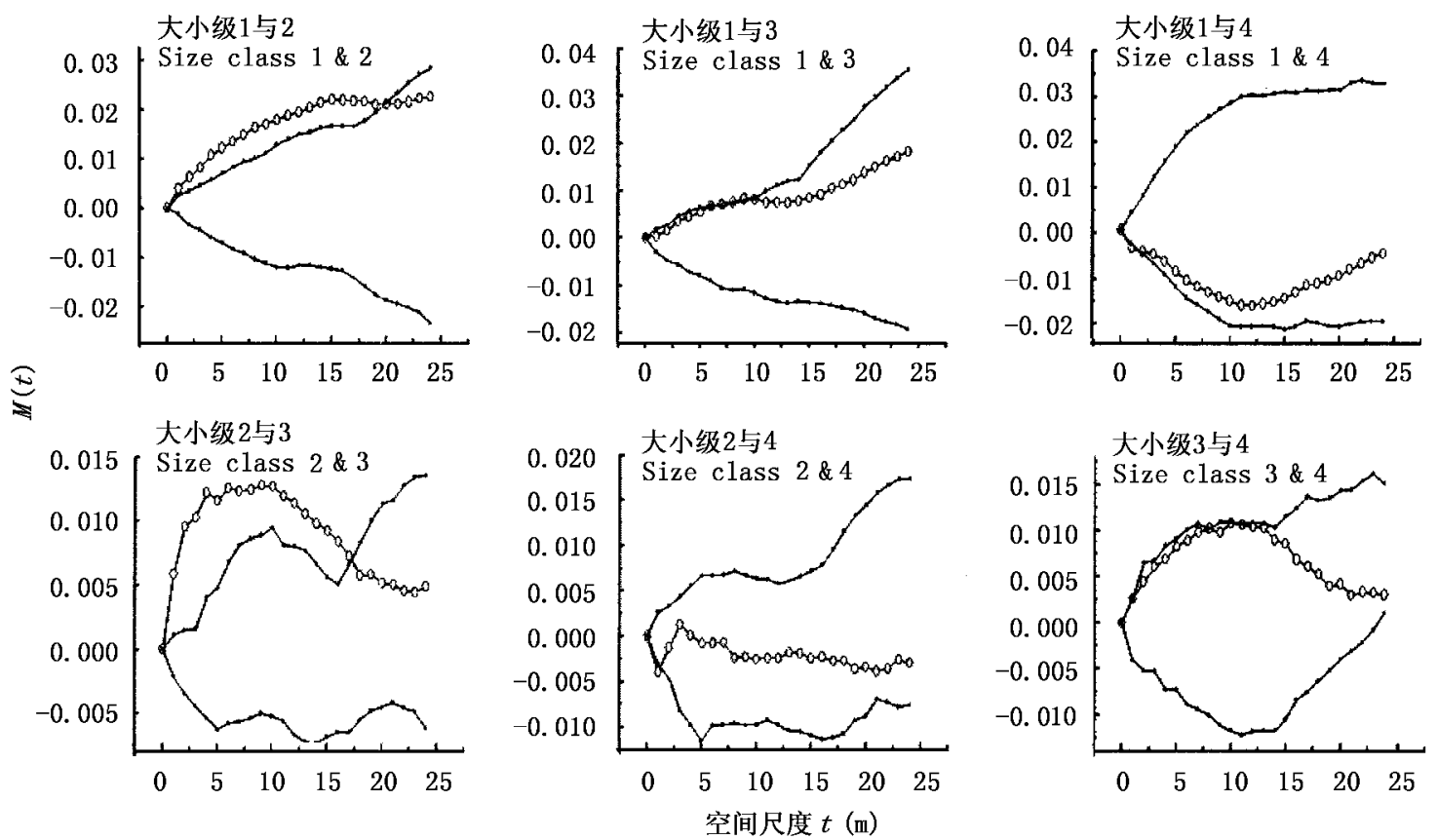

$\rightarrow M(t) \rightarrow$ 上下包迹线 Upper and lower envelopes (95\%)

图 4 半固定沙地油蒿种群任意两个大小级的空间关联

Fig.4 Spatial association of two size classes of Artemisia ordosica population in semi-fixed sandy land

油藁种群的空间格局和空间尺度 (主要指二十 几米内的尺度)有密切关系。在较小的空间尺度上 油蕎种群每个大小级都倾向于非随机分布 (主要是 集群分布, 有时是均匀分布), 当空间尺度大于十几
米或二十几米后, 每个大小级转而倾向于随机分布 ; 与此同时，在较小的空间尺度上各大小级易于表现 为正或负的空间关联,在较大的空间尺度上这些空 间关联趋于减弱。种群的空间分布和空间关联性是 

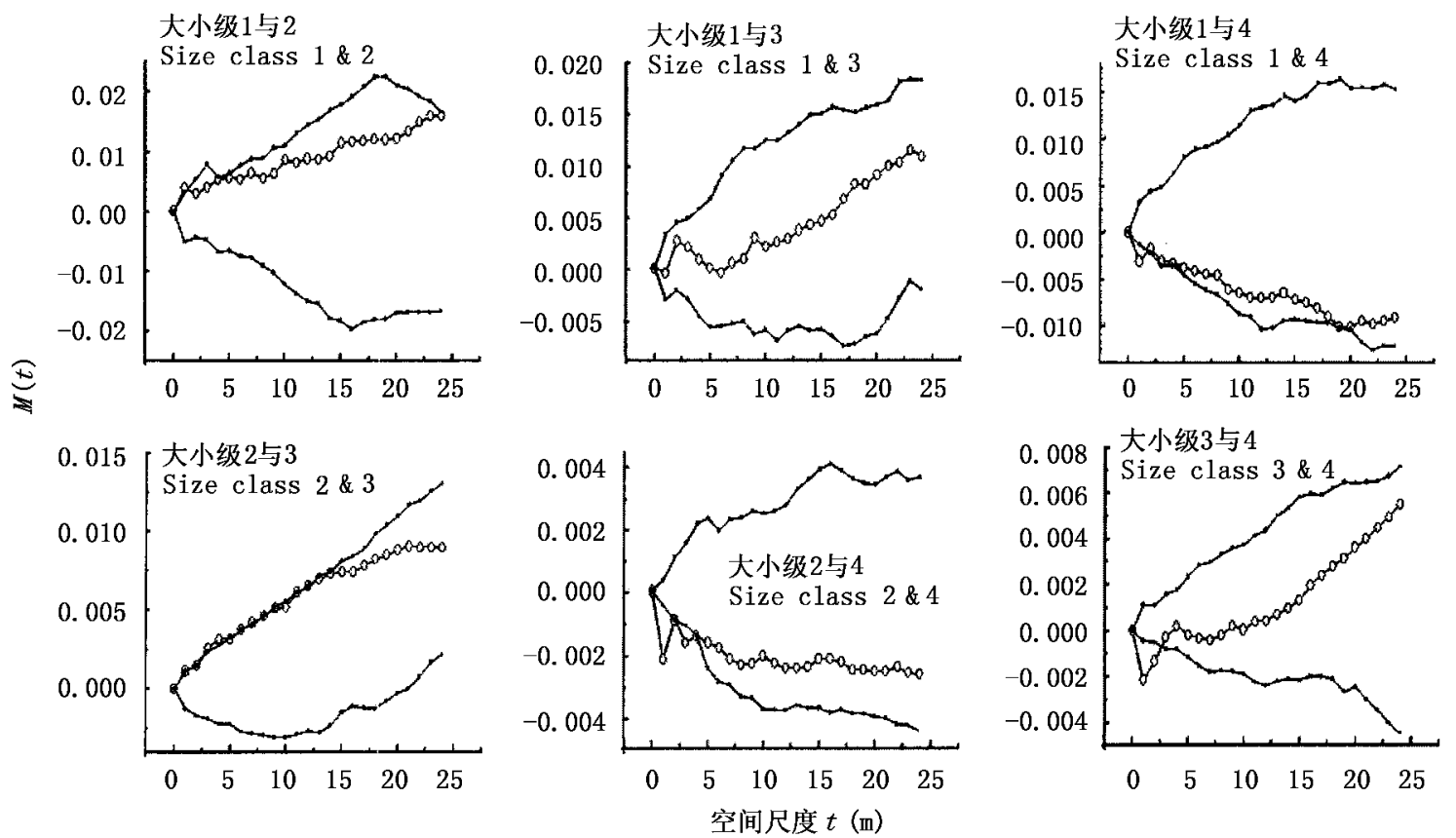

$\infty M(t)$

上下包迹线 Upper and lower envelopes (95\%)

图 5 固定沙地油蕎种群任意两个大小级的空间关联

Fig.5 Spatial association of two size classes of Artemisia ordosica population in fixed sandy land

一致的 (Philips \& Macmahon, 1981; Kenkel, 1988 ; Brission \& Reynolds , 1994 ; Manuel ,2000)。由此可推 断油蓄种群在较小的空间尺度上倾向于非随机分 布, 具有明显的空间关联, 在大于某临界尺度时却倾 向于随机分布, 同时空间关联变得微弱。

油蒿种群的空间格局之所以与空间尺度有这种 联系, 很可能与种群内部关系对空间尺度的依赖性 有关(阳含熙等，1985)。例如，油蕎植株之间关于 土壤水分等生活资源的竞争 种群的个体扩散，以及 个体间为抵御风沙危害的聚集生活和相互庇护等, 可能集中发生在不超过几米到二三十米的距离范围 内, 在这个范围内若相距越近它们的生态联系将越 显著或强烈。其它物种同油蒿的种间关系必然影响 着油䒾种群的空间格局。然而, 由于油蒿是调查样 地的单优建群种, 我们有理由认为它们的空间格局 主要取决于它们的种内关系。

\section{2 形体大小与空间格局的关系}

油蒿植株的形体大小与种群空间格局有密切关 系。随形体增大, 油蕎植株的聚集强度减弱, 甚至有 些大小级在某些尺度上表现为均匀分布 (如固定沙 地油蒿种群的大小级 4 在 1 和 $2 \mathrm{~m}$ 的尺度上表现为 均匀分布)。就油蒿种群的空间关联性而言, 若两个 大小级的形体大小差异越大，它们的空间正关联关 系就越弱,甚至会转变为负关联关系。
通常 幼小个体对水分等资源的竞争相对微弱， 同时为抵御风沙危害和提高存活机会它们需要相互 庇护，于是个体之间以正向的相互联系为主，进而表 现为集群分布 (Allee, 1931 ; 蔡飞, 2000)。反之, 高 大个体对土壤水分或其它资源的竞争会加剧, 同时 由于它们独立抵御风沙危害的能力明显增强, 导致 它们对相互庇护作用的依赖性降低, 结果它们的正 相互联系显著削弱, 同时伴随着聚集强度的降低(李 先琨等,2003)。在结果部分还看到如果两个大小 级的形体大小差异越大，它们的正关联关系就越弱， 甚至表现为负关联关系。据此推测, 油蒿母株产生 的新生个体可能不是集中分布在母株的附近, 而倾 向于分布在与母株保持一定距离的地方, 越是高大 的母株越容易产生排斥。这种现象可能源于种内竞 争的非对称作用，即差异愈显著的植株，它们的竞争 作用对弱小竞争者愈加不利，并导致弱小竞争者的 生长发育迟滞或死亡(侯继华等, 2004)。

\section{3 生境与空间格局的关系}

油葶种群的空间格局与生境亦存在密切关系。 与在固定沙地上相比, 半固定沙地上的油蒿种群及 其各大小级更倾向于集群分布, 同时不同大小级之 间的空间正关联更明显 或者即使存在负关联关系， 也不如在固定沙地上对应大小级之间的负关联关系 明显。这种差别可能与半固定沙地和固定沙地的不 
同的环境条件或风沙干扰程度有关。

在半固定沙地上, 风沙流对植物体的干扰比较 严重, 这会迫使许多个体通过聚集生活抵御风沙流 的危害和提高存活机会; 另一方面, 在半固定沙地上 由于土壤水分条件较好, 降低了油蒿个体之间对土 壤水分这种重要生存条件的竞争(于云江等，2002； 郭柯, 2000)。相反, 在固定沙地上由于风沙危害相 对微弱且土壤水分条件变差, 油蒿个体对聚集生活 的生态需求有所降低，同时水分竞争作用有所增强， 即正向联系减弱和负向联系加强。归纳起来, 油蒿 种群在半固定沙地上表现为较强的空间正关联和集 群分布现象，这很可能是它们应对频繁风沙干扰的 有效生态对策。

\section{4 对植物固沙措施的建议}

我国北方农牧交错带通常以油蕎等耐旱灌木作 为固沙植物, 启动流动沙地的植被发育过程, 最终达 到植被固沙的目的。根据油蓄种群的点格局, 我们 认为当向流动沙地移植这些植物时，应避免将其栽 种成均匀分布或随机分布的形式，而是根据移植个 体的形体大小程度栽种成适当规模的集群分布形 式, 以发挥它们联合抵御风沙危害的能力和提高植 株成活率。

\section{参 考 文 献}

Allee WC (1931). Animal Aggregations: a Study in General Sociology. University of Chicago Press, Chicago.

Andersen M (1992). Spatial analysis of two species interactions. Oecologia, 91, 134-140.

Brisson J, Reynolds JF (1994). The effects of neighbors on root distribution a creosotebush (Larrea tridentata) population. Ecology, 75, $1693-1702$.

Cai F (蔡飞) (2000). A study on the structure and dynamics of Cyclobalanopsis glauca population at hills around west lake in Hangzhou. Scientia Silvae Sinicae (林业科学), 36(3), 67 72. (in Chinese with English abstract)

Dale MRK (1999). Spatial Pattern Analysis in Plant Ecology. Cambridge University Press, Cambridge.

Dale MRT, Powell RD (2001). A new method for characterizing point patterns in plant ecology. Journal of Vegetation Science, 12, $597-608$.

Department of Geography, Peking University (北京大学地理系) (1983). Natural Conditions of the Mu Us Sandy Land and Its Improvement for Utilization (毛乌素沙区自然条件及其改良利 用). Science Press, Beijing. (in Chinese)

Diggle PJ (1983) . Statistical Analysis of Spatial Point Patterns. Academic Press, London.
Greig Smith P (1983). Quantitative Plant Ecology 3rd edn. Blackwell Scientific Publications, Oxford.

Guo K (郭柯) (2000). Cyclic succession of Artemisia ordosic Krasch community in the MU US sandy grassland. Acta Phytoecologica Sinica (植物生态学报), 24, 243 - 247. (in Chinese with English abstract)

Hou JH (侯继华), Huang JH (黄建辉), Ma KP (马克平) (2004). Eleven-year population growth dynamics of major species in a Quercus liaotungensis forest in the Dongling Mountains, northern China. Acta Phytoecologica Sinica (植物生态学 报), 28,609-615. (in Chinese with English abstract)

Huang Z, Gutterman Y (1999). Water absorption by mucilaginous achenes of Artemisia monosperma, floating and germination affected by salt concentrations. Israel Journal of Plant Science, 47, $27-34$.

Kenkel NC (1988). Pattern of self-thinning in Jack pine: testing the random mortality hypothesis. Ecology, 69, 1017 - 1024 .

Li XK (李先琨), Xiang WS (向悟生), Ou ZL (欧祖兰), Su ZM (苏宗明) (2003). Clonal growth spatial pattern and dynamics of the endangered plant Taxus mairei population. Acta Botanica Yunnanica (云南植物研究) , 25, 625-632. (in Chinese with English abstract)

Liu JQ (刘家琼), Li ZJ (黎志坚), Pu JX (蒲锦春), Liu XM (刘新民), Zeng SD (曾泗弟) (1988). Comparative studies on relationships between proline accumulation and photosynthesis, respiration and chlorophyll content of some plant species in the middle part of the desert zone in China. Acta Botanica Sinica (植物学报), 30, 85-89. (in Chinese with English abstract)

Liu ZG (刘振国), Li ZQ (李镇清) (2004). Fine-scale spatial pattern of Artemisia frigida population under different grazing intensities. Acta Ecologica Sinica (生态学报), 24, 227 - 234. (in Chinese with English abstract)

Manly BFJ (1997). Randomization, Bootstrap and Monte-Carlo Methods in Biology 2nd edn. Chapman and Hall, London.

Manuel CM (2000). Ecology: Concept and Application. Science Press \& McGraw-Hill, Beijing.

Philips DL, Macmahon JA (1981). Competition and spacing patterns in desert shrubs. Journal of Ecology, 69, 97 - 115 .

Ripley BD (1977). Modeling spatial patterns. Journal of the Royal Statistical Society (Series B), 39, $172-212$.

Tang MP (汤孟平), Tang SZ (唐守正), Lei XD (雷相东), Zhang HR (张会儒), Hong LX (洪玲霞), Feng YM (冯益 民 ) (2003) . Edge correction of Ripley's $K(d)$ function on population spatial pattern analysis. Acta Ecologica Sinica (生态学 报), 23, 1533-1538. (in Chinese with English abstract)

Wang G (王刚), Liang XG (梁学功) (1995). The dynamics of seed bank on Shapotou artificially stabilized dunes. Acta Botanica Sinica (植物学报), 37, 231-237. (in Chinese with English abstract)

Wang G (王刚), Liang XG (梁学功), Feng B (冯波) (1995). 
germination of Artemisia ordosica, Caragana korshinskii, Hedysarums scoparium. Acta Botanica Boreali-Occidentalia Sinica (西北植物学报), 15(5), 102 - 105. (in Chinese with English abstract)

Wang QS (王庆锁), Dong XJ (董学军), Chen XD (陈旭东), Yang BZ (杨宝珍) (1997). Study on some features of Artemisia ordosica community at the different successional stage. Acta Phytoecologica Sinica (植物生态学报), 21, 531-538. (in Chinese with English abstract)

Yang HX (阳含熙), Li DJ (李鼎甲), Wang BN (王本楠), Han JX (韩进轩) (1985). Distribution patterns of the main tree-species of broad-leaved Korean-pine forests on the north slope of Changbai Mountains (长白山北坡阔叶红松林主要树 种的分布格局). Research of Forest Ecosystems (森林生态系 统研究) , 5, 1-14. (in Chinese)

Yang HX (杨洪晓), Zhang JT (张金屯), Wu B (吴波), Wang Y (王妍), Li XS (李晓松), Xu B (许彬) (2004). Adaptation of Artemisia ordosica to temperate arid sandy land and its roles in habitat shift. Journal of Beijing Normal University (北京 师范大学学报), 40,684-689. (in Chinese with English ab- stract)

Yu YJ (于云江), Lin QG (林庆功), Shi QH (石庆辉), Liu JQ (刘家琼) (2002). Changes of habitat and vegetation in manmade vegetation area of Shapotou section along Baotou-Lanzhou railway. Acta Ecologica Sinica (生态学报), 22, 433 - 439. (in Chinese with English abstract)

Zhang JT (张金屯) (1998). Analysis of spatial point pattern for plant species. Acta Phytoecologica Sinica (植物生态学报), 22, 344 - 349. (in Chinese with English abstract)

Zhang JT (张金屯) (2004). Quantitative Ecology (数量生态 学). Science Press, Beijing. (in Chinese)

Zhang JT (张金屯), Meng DP (孟东平) (2004). Spatial pattern analysis of individuals in different age-classes of Larix principisrupprechtii in Luya mountain reserve, Shanxi, China. Acta Ecologica Sinica (生态学报), 24, 35-40. (in Chinese with English abstract)

Zhang XS (张新时) (1994). Principles and optimal models for development of Maowusu sandy grassland. Acta Phytoecologica Sini$c a$ (植物生态学报), 18, 1-16. (in Chinese with English abstract) 